\title{
Inner-product spaces for quantitative analysis of eyes and other optical systems
}

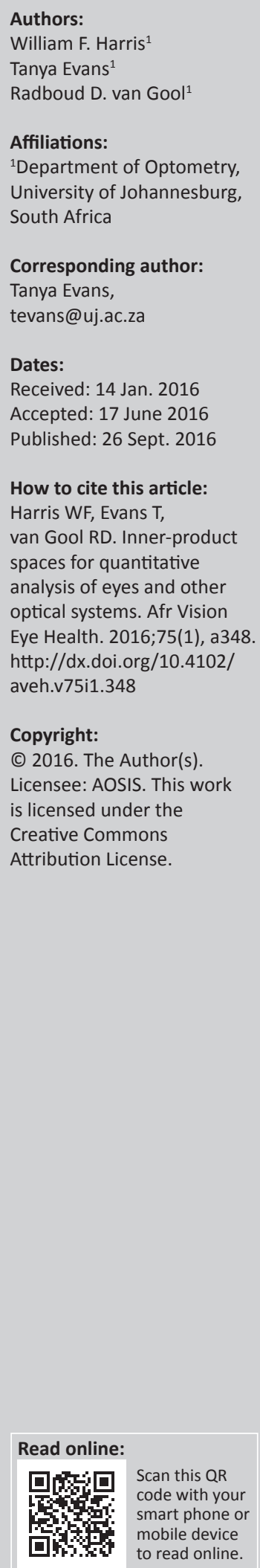

Because dioptric power matrices of thin systems constitute a (three-dimensional) inner-product space, it is possible to define distances and angles in the space and so do quantitative analyses on dioptric power for thin systems. That includes astigmatic corneal powers and refractive errors. The purpose of this study is to generalise to thick systems. The paper begins with the ray transference of a system. Two 10-dimensional inner-product spaces are devised for the holistic quantitative analysis of the linear optical character of optical systems. One is based on the point characteristic and the other on the angle characteristic; the first has distances with the physical dimension $\mathrm{L}^{-1}$ and the second has the physical dimension L. A numerical example calculates the locations, distances from the origin and angles subtended at the origin in the 10-dimensional space for two arbitrary astigmatic eyes.

\section{Introduction}

The optical character of a thin system in linear optics can be represented by a symmetric $2 \times 2$ matrix F, the symmetric dioptric power matrix. The set of all such powers defines a threedimensional linear (or vector) space, known as symmetric dioptric power space. ${ }^{1}$ Because the matrix has uniform physical dimensionality ${ }^{2}$ (each entry has the dimension $\mathrm{L}^{-1}$ and is usually measured in dioptres), one can define an inner-product on the space and the space becomes an inner-product space. Because symmetric dioptric power space is an inner-product space, we have been able to define distances, angles, orthonormal axes, confidence ellipsoids, etc. in the space. This has provided the basis for the quantitative analysis we have done on powers including refractive errors and corneal powers (e.g. Ref 3).

For some years, we have sought to extend this type of analysis to thick systems such as the eye (e.g. Ref 4). In linear optics, the optical character of a system that is thick or thin is completely characterised by the ray transference (a real $4 \times 4$ matrix)

$\mathbf{S}=\left(\begin{array}{ll}\mathbf{A} & \mathbf{B} \\ \mathbf{C} & \mathbf{D}\end{array}\right)$

of the system. ${ }^{5}$ In strong contrast to the set of symmetric dioptric powers, the set of transferences is neither a linear space nor does it have uniform dimensionality. Therefore, there is no innerproduct space that would provide a basis for holistic quantitative analysis of the optical character of thick systems such as the eye. The purpose of this study is to show how inner-product spaces can in fact be constructed for general optical systems.

\section{Method}

The method is based on the transference. The transference $\mathbf{S}$ (Equation 1) obeys the following equation ${ }^{5}$

$\mathbf{S}^{\mathrm{T}} \mathbf{E S}=\mathbf{E}$

where

$\mathbf{E}=\left(\begin{array}{cc}\mathbf{O} & \mathbf{I} \\ -\mathbf{I} & \mathbf{O}\end{array}\right)$

and $\mathbf{I}$ and $\mathbf{O}$ are identity and null matrices, respectively. Such matrices are called symplectic. ${ }^{6} \mathbf{A}, \mathbf{B}$, $\mathbf{C}$ and $\mathbf{D}$ are $2 \times 2$ submatrices of $\mathbf{S}$ and represent the fundamental (linear) optical properties of the system. ${ }^{7} \mathbf{B}$ has the physical dimension $\mathrm{L}$ and $\mathbf{C}$ the physical dimension $\mathrm{L}^{-1}$; the other two fundamental 
properties are dimensionless. Other optical properties of the system can be obtained from the fundamental properties; for example, the power of the system is given $\mathrm{by}^{7}$

$\mathbf{F}=-\mathbf{C}$

[Eqn 4]

and, for eyes, the corneal-plane refractive compensation (or refractive error) is given $\mathrm{by}^{7}$

$\mathbf{F}_{0}=\mathbf{B}^{-1} \mathbf{A}$.

[Eqn 5]

Two matrices related to the transference are the point characteristic

$\mathbf{P}=\left(\begin{array}{cc}\mathbf{B}^{-1} \mathbf{A} & -\mathbf{B}^{-1} \\ -\mathbf{B}^{-\mathrm{T}} & \mathbf{D B}^{-1}\end{array}\right)=\left(\begin{array}{cc}\mathbf{U} & \mathbf{V} \\ \mathbf{V}^{\mathrm{T}} & \mathbf{W}\end{array}\right)$

[Eqn 6]

and the angle characteristic

$\mathbf{Q}=\left(\begin{array}{cc}\mathbf{C}^{-1} \mathbf{D} & \mathbf{C}^{-1} \\ \mathbf{C}^{-\mathrm{T}} & \mathbf{A C}^{-1}\end{array}\right)=\left(\begin{array}{cc}\mathbf{X} & \mathbf{Y} \\ \mathbf{Y}^{\mathrm{T}} & \mathbf{Z}\end{array}\right)$

Elsewhere $^{8}$ we use these matrices to calculate average systems.

\section{Results}

From $\mathbf{P}$ and $\mathbf{Q}$ we construct the $2 \times 6$ matrices

$\mathbf{G}=\left(\begin{array}{lll}\mathbf{U} & \mathbf{V} & \mathbf{W}\end{array}\right)$

[Eqn 8]

and

$\mathbf{H}=\left(\begin{array}{lll}\mathbf{X} & \mathbf{Y} & \mathbf{Z}\end{array}\right)$.

[Eqn 9]

It is a consequence of symplecticity (Equation 2) that $\mathbf{U}, \mathbf{W}$, $\mathbf{X}$ and $\mathbf{Z}$ are symmetric; $\mathbf{V}$ and $\mathbf{Y}$ are general. (Properties of symplectic matrices are summarised elsewhere. ${ }^{9}$ ) The set of all matrices $G$ is a linear space and $G$ has uniform physical dimensionality $\left(\mathrm{L}^{-1}\right)$. Similarly matrices $\mathbf{H}$ define a dimensionally uniform (dimension L) linear space.

G can be expanded as

$$
\begin{aligned}
\mathbf{G}= & U_{\mathbf{I}}\left(\begin{array}{lll}
\mathbf{I} & \mathbf{O} & \mathbf{O}
\end{array}\right)+U_{\mathbf{J}}\left(\begin{array}{lll}
\mathbf{J} & \mathbf{O} & \mathbf{O}
\end{array}\right)+U_{\mathbf{K}}\left(\begin{array}{lll}
\mathbf{K} & \mathbf{O} & \mathbf{O}
\end{array}\right) \\
& +V_{\mathbf{I}}\left(\begin{array}{lll}
\mathbf{O} & \mathbf{I} & \mathbf{O}
\end{array}\right)+V_{\mathbf{J}}\left(\begin{array}{lll}
\mathbf{O} & \mathbf{J} & \mathbf{O}
\end{array}\right)+V_{\mathbf{K}}\left(\begin{array}{lll}
\mathbf{O} & \mathbf{K} & \mathbf{O}
\end{array}\right)+V_{\mathbf{L}}\left(\begin{array}{lll}
\mathbf{O} & \mathbf{L} & \mathbf{O}
\end{array}\right) \\
& +W_{\mathbf{I}}\left(\begin{array}{lll}
\mathbf{O} & \mathbf{O} & \mathbf{I}
\end{array}\right)+W_{\mathbf{J}}\left(\begin{array}{lll}
\mathbf{O} & \mathbf{O} & \mathbf{J}
\end{array}\right)+W_{\mathbf{K}}\left(\begin{array}{lll}
\mathbf{O} & \mathbf{O} & \mathbf{K}
\end{array}\right)
\end{aligned}
$$

where

$\mathbf{J}=\left(\begin{array}{cc}1 & 0 \\ 0 & -1\end{array}\right), \mathbf{K}=\left(\begin{array}{ll}0 & 1 \\ 1 & 0\end{array}\right)$ and $\mathbf{L}=\left(\begin{array}{cc}0 & 1 \\ -1 & 0\end{array}\right)$
Also,

$$
\begin{array}{cc}
V_{\mathbf{I}}=\left(v_{11}+v_{22}\right) / 2 & V_{\mathbf{J}}=\left(v_{11}-v_{22}\right) / 2 \\
V_{\mathbf{K}}=\left(v_{12}+v_{21}\right) / 2 & V_{\mathbf{L}}=\left(v_{12}-v_{21}\right) / 2
\end{array}
$$

and similarly for $U_{\mathrm{I}}$ and the other coefficients in Equation 10. We define the coordinate vector

$$
\mathbf{g}=\left(\begin{array}{llllllllll}
U_{\mathbf{I}} & U_{\mathbf{J}} & U_{\mathbf{K}} & V_{\mathbf{I}} & V_{\mathbf{J}} & V_{\mathbf{K}} & V_{\mathbf{L}} & W_{\mathbf{I}} & W_{\mathbf{J}} & W_{\mathbf{K}}
\end{array}\right)^{\mathrm{T}}
$$

[Eqn 12]

relative to the basis

$$
\beta=\left\{\left(\begin{array}{lll}
\mathbf{I} & \mathbf{O} & \mathbf{O}
\end{array}\right),\left(\begin{array}{lll}
\mathbf{J} & \mathbf{O} & \mathbf{O}
\end{array}\right),\left(\begin{array}{ccc}
\mathbf{K} & \mathbf{O} & \mathbf{O}
\end{array}\right), \cdots,\left(\begin{array}{lll}
\mathbf{O} & \mathbf{O} & \mathbf{K}
\end{array}\right)\right\} .
$$

Consider two optical systems 1 and 2. Their coordinate vectors are $\mathbf{g}_{1}$ and $\mathbf{g}_{2}$. Now we define the inner-product of $\mathbf{g}_{1}$ and $\mathbf{g}_{2}$ by

$$
\left\langle\mathbf{g}_{1}, \mathbf{g}_{2}\right\rangle=\mathbf{g}_{1}^{\mathrm{T}} \mathbf{g}_{2}
$$

Consequently we have distances (magnitudes) $g$ and angles $\theta$ in the space defined by

$$
g=\sqrt{\mathbf{g}^{\mathrm{T}} \mathbf{g}}
$$

and

$$
\cos \theta=\frac{\mathbf{g}_{1}^{\mathrm{T}} \mathbf{g}_{2}}{g_{1} g_{2}}
$$

respectively.

Thus we have a 10-dimensional inner-product space for quantitative analysis of optical systems in linear optics for which B is non-singular. One can think of distances in the space as powers (e.g. dioptres).

For matrices of the form $\mathbf{H}$ (Equation 9), one can follow a similar approach. It leads to a second 10-dimensional innerproduct space. It applies for optical systems for which $\mathrm{C}$ is non-singular and distances in the space are lengths (e.g. metres).

We illustrate the theory using two optical systems whose transferences have been presented before: ${ }^{8}$

$$
\mathbf{S}_{1}=\left(\begin{array}{cccc}
-0.2066 & -0.0031 & 0.0200 & 0.0000 \\
-0.0031 & -0.2240 & 0.0000 & 0.0200 \\
-58.8160 & -0.0853 & 0.8569 & 0.0017 \\
-0.0841 & -59.5090 & 0.0017 & 0.8599
\end{array}\right)
$$


and

$\mathbf{S}_{2}=\left(\begin{array}{cccc}-0.1641 & 0.0060 & 0.0197 & 0.0000 \\ 0.0060 & -0.1399 & 0.0000 & 0.0197 \\ -57.9190 & 0.3455 & 0.8670 & 0.0024 \\ 0.3415 & -56.9734 & 0.0024 & 0.8637\end{array}\right)$,

the units being dioptres and metres. The coordinate vectors (Equation 12) turn out to be

$$
\begin{gathered}
\mathbf{g}_{1}=\left(\begin{array}{ccccccc}
-10.7650 & 0.4350 & -0.1550 & -50.0000 & 0 & 0 & 0 \\
42.9200 & -0.0750 & 0.0850
\end{array}\right)^{\mathrm{T}} \mathrm{D}
\end{gathered}
$$

and

$$
\begin{aligned}
\mathbf{g}_{2}=\left(\begin{array}{lllllll}
-7.7157 & -0.6142 & 0.3046 & -50.7614 & 0 & 0 & 0 \\
43.9264 & 0.0838 & 0.1218
\end{array}\right)^{\mathrm{T}} \mathrm{D} &
\end{aligned}
$$

These vectors locate the two optical systems relative to the origins of the space. Their distances from the origin are $g_{1}=66.77 \mathrm{D}$ and $g_{2}=67.57 \mathrm{D}$, respectively, and they subtend an angle $\theta=2.90^{\circ}$ at the origin.

\section{Conclusion}

We have here constructed two inner-product spaces for the linear optical characters of optical systems. One is based on the point characteristic and the other on the angle characteristic. Both spaces can be used for eyes because they have non-singular $\mathbf{B}$ and $\mathbf{C}$. We now have the machinery for holistic quantitative analysis of optical systems in general and eyes in particular.

\section{Acknowledgements}

W.F.H. acknowledges support from the National Research Foundation of South Africa. This work was presented as a poster presentation ${ }^{10}$ at Visual and Physiological Optics 2014 conference in Wrocław, Poland.

\section{Competing interests}

The authors declare that they have no financial or personal relationships which may have inappropriately influenced them in writing this article.

\section{Authors' contributions}

The work was a team effort led by W.F.H. with contributions from T.E. and R.D.v.G. over several years.

\section{References}

1. Harris WF. Representation of dioptric power in Euclidean 3-space. Ophthalmic Physiol Opt. 1991;11:130-136. http://dx.doi.org/10.1111/j.1475-1313.1991. tb00212.x

2. Hart GW. Multidimensional analysis: Algebras and systems for science and engineering. New York: Springer, 1995; p. 66.

3. Harris WF. Reduction of artefact in scatter plots of spherocylindrical data. Ophthalmic Physiol Opt. 2005;25:13-17. http://dx.doi.org/10.1111/j.1475-1313. 2004.00249.x

4. Harris WF, Cardoso JR. The exponential-mean-log-transference as a possible representation of the optical character of an average eye. Ophthalmic Physiol Opt. 2006;26:380-383. http://dx.doi.org/10.1111/j.1475-1313.2006.00365.x

5. Torre A. Linear ray and wave optics in phase space. Volume 60. Amsterdam Elsevier, 2005; p. 60, 155

6. Guillemin V, Sternberg S. Symplectic techniques in physics. Cambridge: Cambridge University Press; 1984; 23 et seq.

7. Harris WF. Dioptric power: Its nature and its representation in three- and four-dimensional space. Optom Vis Sci. 1997;74:349-366.

8. van Gool RD, Harris WF. The concept of the average eye. S Afr Optom. 2005; 64:38-43.

9. Harris WF. Symplecticity and relationships among the fundamental properties in linear optics. S Afr Optom. 2010;69:3-13.

10. Harris WF, Evans T, van Gool RD. Inner-product spaces for quantitative analysis of eyes and other optical systems. Proceedings of the VII European/I World Meeting in Visual and Physiological Optics VPOptics, 2014 August 25-27, Wrocław, Poland, Volume 7, ed by DR Iskander and HT Kasprzak. 2014; pp. 116-119. 